\title{
Helium Abundance in the Most Metal-Deficient Dwarf Galaxies
}

\author{
Y. I. Izotov \\ Main Astronomical Observatory, Goloseevo, Kiev-22, 252650 Ukraine
}

\begin{abstract}
The high-quality long-exposure spectroscopic observations of the two most-metal deficient blue compact galaxies I $\mathrm{Zw} 18$ and SBS 0335-052 are discussed. We confirm previous findings that underlying stellar absorption strongly influences the observed intensities of He I emission lines in the brightest $\mathrm{NW}$ component of $\mathrm{I} \mathrm{Zw} 18$, and hence this component should not be used for primordial He abundance determination. The effect of underlying stellar absorption, though present, is much smaller in the $\mathrm{SE}$ component. The extremely high signal-to-noise ratio spectrum ( $\geq 100$ in the continuum) of the BCG SBS $0335-052$ allows us to measure the helium mass fraction with precision better than $2 \%$ in nine different regions along the slit. The weighted mean of helium mass fraction in two most metal-deficient BCGs I Zw 18 and SBS 0335-052, $Y=0.2462 \pm 0.0009$, after correction for the He production in massive stars results in primordial He mass fraction $Y_{p}=0.2452 \pm 0.0009$.
\end{abstract}

\section{Introduction}

Blue compact galaxies (BCGs) are ideal objects for the determination of primordial helium abundance and hence for determination of one of the fundamental cosmological parameters - baryon mass fraction in the Universe.

One of the important questions is how robust are measurements of He abundance in BCGs. We discuss this problem using new highest signal-to-noise ratio observations of two most metal-deficient BCGs known, I Zw 18 and SBS 0335052 . Due to the very low oxygen abundances $\left(Z_{\odot} / 50\right.$ and $Z_{\odot} / 40$ in $\mathrm{I} \mathrm{Zw} 18$ and SBS 0335-052 respectively) the helium mass fraction in these galaxies is very close to the primordial value $Y_{p}$ which we derive in this paper as mean value in two galaxies.

\section{Observations}

Spectrophotometric observations of I $\mathrm{Zw} 18$ were obtained with the Multiple Mirror Telescope (MMT) on the nights of 1997 April 29 and 30. The total exposure time was 180 minutes and was broken up into six sub-exposures, 30 minutes each. The slit was oriented in the direction with position angle P.A. $=-41^{\circ}$ to permit observations of both NW and SE components. The Keck II telescope optical spectra of SBS 0335-052 were obtained on 1998 February 24 with Low Resolution Imaging Spectrometer (Izotov et al. 1998). The slit 

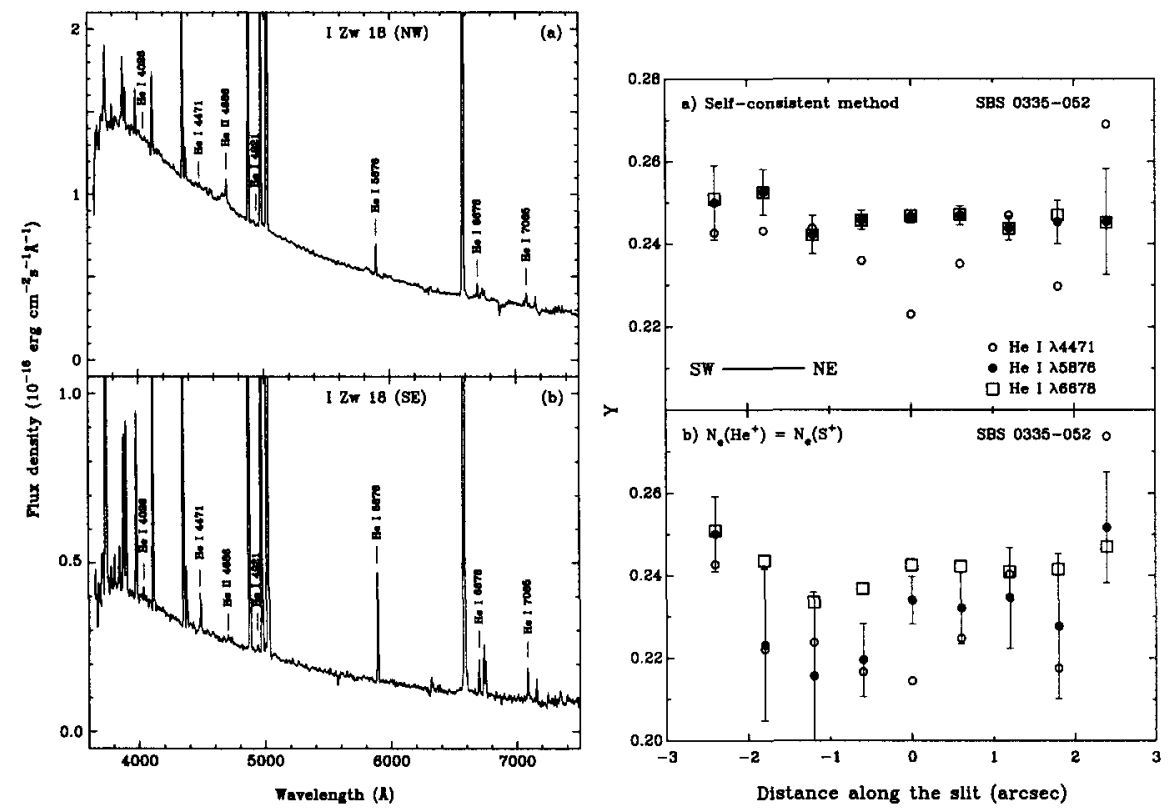

Figure 1. Left panel: The MMT spectra of brightest parts of the NW and the SE components of the I Zw 18. Note that all marked He I lines in the spectrum of the SE component are in emission while two He I $\lambda 4026$ and $\lambda 4921$ lines are in absorption in the spectrum of the NW component. Right panel: The spatial distributions of the helium mass fractions in the SBS 0335-052. The helium mass fractions (upper plot) are derived self-consistently from the observed He I $\lambda 3889$, $\lambda 4471, \lambda 5876, \lambda 6678$ and $\lambda 7065$ emission line intensities. The helium mass fractions in lower plot are derived from the He I line intensities corrected only for collisional enhancement with electron number density $N_{e}$ (S II).

was oriented in direction with position angle P.A. $=60^{\circ}$ perpendicular to the SBS 0335-052 major axis. The total exposure time was $40 \mathrm{~min}$, broken to two exposures of $30 \mathrm{~min}$ and $10 \mathrm{~min}$.

In Figure 1a (left panel) we show one-dimensional spectrum of the NW component of I Zw 18 in its brightest part with aperture $0.6^{\prime \prime} \times 1.5^{\prime \prime}$ which shows broad WR bumps at $\lambda 4650$ and $\lambda 5808$ and which have been discussed by Izotov et al. (1997). In Figure 1b (left panel) the spectrum of SE component in aperture $0.6^{\prime \prime} \times 1.5^{\prime \prime}$ is shown at the angular distance $5.4^{\prime \prime}$ from the NW component. All He I lines in the spectrum of the SE component are in emission while two He I $\lambda 4026$ and $\lambda 4921$ lines are in absorption and He I $\lambda 4471$ emission line is barely seen in the spectrum of the NW component. Other three He I lines marked in the spectrum of the NW component are in emission, although their intensities are reduced due to the presence of underlying stellar absorption. 
Thanks to the very highest signal-to-noise ratio Keck II spectrum of SBS 0335-052 with SNR $\geq 100$ for the continuum in its brightest part, several apertures have been applied for extraction of one-dimensional spectra. We report here the discovery of the weak WR bump, which is detected only in the brightest part of SBS 0335-052. Hence Wolf-Rayet stars are present in two most metal-deficient galaxies I Zw 18 and SBS 0335-052.

\section{Helium Abundance}

To derive element abundances, we have followed the procedure detailed by Izotov, Thuan \& Lipovetsky $(1994,1997)$. He emission-line strengths are converted to singly ionized helium $y^{+} \equiv \mathrm{He}^{+} / \mathrm{H}^{+}$and doubly ionized helium $y^{++} \equiv$ $\mathrm{He}^{++} / \mathrm{H}^{+}$. The main mechanisms deviating $\mathrm{He} \mathrm{I}$ emission line intensities from the recombination values are collisional and fluorescent enhancements. In order to correct for these effects, we have adopted the following procedure: we have evaluated the electron number density $N_{e}$ (He II) and the optical depth $\tau(\lambda 3889)$ in the He I $\lambda 3889$ line in a self-consistent way, so that the He I $\lambda 3889 / \lambda 4471$, $\lambda 5876 / \lambda 4471, \lambda 6678 / \lambda 4471$ and $\lambda 7065 / \lambda 4471$ line ratios have their recombination values, after correction for collisional and fluorescent enhancements. The very high signal-to-noise ratio Keck II observations of SBS 0335-052 allow us to derive with great precision the helium mass fraction in nine different regions in this BCG. We find that both collisional and fluorescent enhancements of $\mathrm{He}$ I emission lines are important in SBS 0335-052 and should be taken into account properly. It is shown (Figure 1, right panel) that electron number density derived from [S II] emission lines cannot be used due to the overcorrection of the $\mathrm{He} \mathrm{I}$ emission line intensities for said effects by $5-10 \%$ and consequently to underestimation of $\mathrm{He}$ mass fraction in this BCG. When the self-consistent method is used the helium mass fractions $Y$ derived from He I $\lambda 5876$ and $\lambda 6678$ emission line intensities are in perfect agreement in each of 9 regions while the He mass fraction derived from He I $\lambda 4471$ emission line is systematically lower due to the presence of underlying stellar absorption. The weighted mean of He mass fraction for all 9 regions of SBS $0335-052$ is $Y=0.2463 \pm 0.0009$ if He I $\lambda 5876$ and $\lambda 6678$ emission lines are used. This $Y$ value leads to primordial value $Y_{p}=$ $0.2453 \pm 0.0009$, after correction for He enrichment by massive stars, and corresponds to baryon-to-photon number ratio $\eta=(4.7 \pm 0.4) \times 10^{-10}$ which translates to baryon mass fraction $\Omega_{b} h_{50}^{2}=0.068 \pm 0.006\left(h_{50}=H_{0} / 50 \mathrm{~km} \mathrm{~s}^{-1} \mathrm{Mpc}^{-1}\right)$.

\section{References}

Izotov, Y. I., Chaffee, F. H., Foltz, C. B., Green, R. F., \& Guseva, N. G. 1998, in preparation

Izotov, Y. I., Foltz, C. B., Green, R. F., Guseva, N. G., \& Thuan, T. X. 1997, ApJ, 487, L37

Izotov, Y. I., \& Thuan, T. X. 1998, ApJ, 497, 227

Izotov, Y. I., Thuan, T. X., \& Lipovetsky, V. A. 1994, ApJ, 435, 647

Izotov, Y. I., Thuan, T. X., \& Lipovetsky, V. A. 1997, ApJS, 108, 1 